\title{
Stable Droplets and Dark-Ring Cavity Solitons in Nonlinear Optical Devices
}

\author{
Damià Gomila, Pere Colet, Maxi San Miguel, Andrew J. Scroggie, and Gian-Luca Oppo
}

\begin{abstract}
Two kinds of cavity solitons, stable circular domain walls (droplets) and dark-ring cavity solitons, are presented in models of vectorial Kerr resonators and degenerate optical parametric oscillators. These structures are universal in systems with two equivalent homogeneous states and are found for parameter values close to those of a modulational instability of a flat front. Stable droplets owe their existence to curvature effects and, therefore, they are not present in one-dimensional systems. We show that stable droplets nucleate out of dark-ring cavity solitons and that in some systems there are regimes in which they coexist.
\end{abstract}

Index Terms-Cavity solitons, optical parametric oscillators, vectorial Kerr resonators.

\section{INTRODUCTION}

$\mathbf{S}_{\mathrm{P}}^{\mathrm{p}}$ PATIALLY localized structures appearing in the transverse plane of optical cavities filled with quadratic or cubic nonlinear media have attracted a lot of attention in recent years [1]. In particular, cavity solitons in regimes where a homogeneous and a spatially modulated solution coexist and are stable have been discovered and shown to be useful for optical coding and memory applications [2], [3]. A related yet different class of cavity solitons has been associated with the existence and equal stability of two homogeneous solutions (also called phases) which differ from each other by a $\pi$ phase shift in the slowly varying amplitude of the electric field or by their polarization state. Systems which satisfy the latter condition are commonplace in optics due to either the polarization symmetry or the quadratic nature of the nonlinearity. The cavity solitons in these systems in two transverse dimensions are often formed by shrinking domains of one phase embedded in the other. The domain walls separating the two equivalent phases are narrow spatial features [4] and present damped oscillations

Manuscript received June 14, 2002; revised September 16, 2002. This work was supported by the EC Networks QSTRUCT (FMRXCT960077) and QUANTIM (IST-2000-26019), and by the European Science Foundation network PHASE. The work of D. Gomlia, P. Colet, and M. San Miguel was supported by MCyT, Spain, under Project PB97-0141-C02-02 and Project BFM2000-1108. The work of A. J. Scroggie and G.-L. Oppo was supported by the EPSRC under Grant M19727, Grant M31880, and Grant R04096. The work of G.-L. Oppo was supported in part by SGI.

D. Gomila, P. Colet, and M. San Miguel are with the Instituto Mediterráneo de Estudios Avanzados, IMEDEA, Campus Universitat Illes Balears, E-07071 Palma de Mallorca, Spain (e-mail address: gomila@imedea.uib.es).

A. J. Scroggie and G.-L. Oppo are with the Department of Physics, University of Strathclyde, Glasgow G4 ONG, Scotland, U.K. (e-mail address: gianluca@ phys.strath.ac.uk).

Digital Object Identifier 10.1109/JQE.2002.807209 in their tails due to diffraction. When a domain of one phase embedded in the other shrinks, these oscillatory tails interact and may form stable localized states. The intensity profile of these cavity solitons is characterized by a peak surrounded by a dark ring and then by a homogeneous phase. Dark-ring cavity solitons (DRCS) have been described first in the mathematical context of the Swift-Hohenberg equation [5] and later in the degenerate optical parametric oscillator (DOPO) both off [6] and at resonance [7], [8], and in the vectorial Kerr resonator [9]. These are the two nonlinear optical systems discussed here.

Recently, we have also described the existence of a novel kind of stable localized structure in a generic model equation, the parametrically driven Ginzburg-Landau equation: the stable droplet [10]. In contrast with DRCS, stable droplets are large stable circular domain walls separating the two homogeneous solutions. Stable droplets have been shown to appear close to the modulational instability of a flat domain wall and balance the curvature-driven shrinking of a domain with the growth due to the instability of tightly curved fronts [10]. There is a fundamental difference between the mechanism that allows for the existence of previously reported localized structures, namely, cavity solitons in regimes where a homogeneous and a spatially modulated solution coexist, and cavity solitons associated with the existence of two homogeneous solutions (DRCS), and the stable droplet. While the first two types of cavity solitons exist both in one- and two-dimensional systems, stable droplets do not exist in one dimension because their stability is due to curvature effects. Therefore, they only exist in systems whose dimensionality is at least two. It is the main aim of this paper to show that stable droplets appear rather generally in nonlinear optical devices. We study the details of stable droplets in a vectorial Kerr resonator and DOPO and we characterize the transition from DRCS to stable droplet in those systems.

\section{General Theory of Stable Droplets}

Before entering into the details of the models of the nonlinear optical devices, we recall the main points of the theory behind the existence and stability of stable droplets close to the modulational instability of a flat front [10]. In particular, we consider systems with two different homogeneous solutions which are equivalent under symmetries of the system. For the systems we consider, and for the case of transverse dimensionality $d=1$, a front connecting the two states does not move since they are 
equivalent. However, for $d=2$, these fronts may move due to curvature effects. For simplicity, let us consider the movement of a domain with circular symmetry. The radius of a circular domain of one solution embedded in the other evolves in time as

$$
\dot{R}(t)=-\gamma\left(E_{0}\right) / R
$$

where $E_{0}$ is a control parameter of the system, typically, the pump field in nonlinear optical cavities. The coefficient $\gamma$ can be calculated from the profile of the one-dimensional $(d=1)$ front connecting the two equivalent states [10]. The profile of the front depends on the control parameter $E_{0}$ and, therefore, the coefficient $\gamma$ depends also on $E_{0}$ through the profile of the front. For a large variety of systems, the coefficient $\gamma$ can change sign upon variations of the control parameter. We identify the value $E_{0}=E_{0,1}$ of the control parameter for which $\gamma$ vanishes, and we assume $\gamma>0(\gamma<0)$ for $E_{0}>E_{0,1}\left(E_{0}<E_{0,1}\right)$. When $\gamma$ is positive and large, any circular phase domain with arbitrary initial radius shrinks until it disappears. It is observed that arbitrarily shaped domains also shrink and disappear and the typical domain size decreases as in (1). If $\gamma$ is negative, any circular domain will grow due to curvature effects. In addition, any perturbation of a wall grows so that in this regime a flat wall is modulationally unstable and a generic initial condition evolves into labyrinthine patterns. Therefore, $E_{0,1}$ signals the place at which a flat wall connecting the two equivalent states becomes modulationally unstable.

Close to $E_{0,1}, \gamma$ can be written as $c_{1}\left(E_{0}-E_{0,1}\right)$ and nonlinear curvature corrections have to be taken into account. In this case, an amplitude equation for the curvature $\kappa$ of gently curved fronts can be derived rather generally [10]. In the case of circular domains of radius $R, \kappa=1 / R$, and the following equation for the radius is obtained [10]:

$$
\dot{R}=-c_{1}\left(E_{0}-E_{0,1}\right) \frac{1}{R}-c_{3} \frac{1}{R^{3}}
$$

where the coefficients $c_{1}$ and $c_{3}$ can be calculated from the profile of the $d=1$ front connecting the two equivalent solutions at $E_{0}=E_{0,1}$ following the procedure indicated in [10]. Since we are considering $\gamma>0$ for $E_{0}>E_{0,1}$, it follows that $c_{1}>0$. If $c_{3}$ is negative (supercritical bifurcation), our analysis predicts just above $E_{0,1}$ the existence of stable stationary circular domains (the stable droplet) with a radius $R_{0}$ given by

$$
R_{0}=\frac{1}{\sqrt{E_{0}-E_{0,1}}} \sqrt{\frac{-c_{3}}{c_{1}}} .
$$

For $E_{0}$ larger than but close to $E_{0,1}$, an initially small (very large) domain grows (shrinks) until a stable droplet is formed. Note that the radius of the stable droplet diverges at $E_{0,1}$.

Finally, we demonstrated in [10] that domains of arbitrary shape evolve first to circular domains and then to stable droplets, making the stable droplet an attractor and the most relevant localized structure for values of the control parameter just above the modulational instability of the flat domain wall.

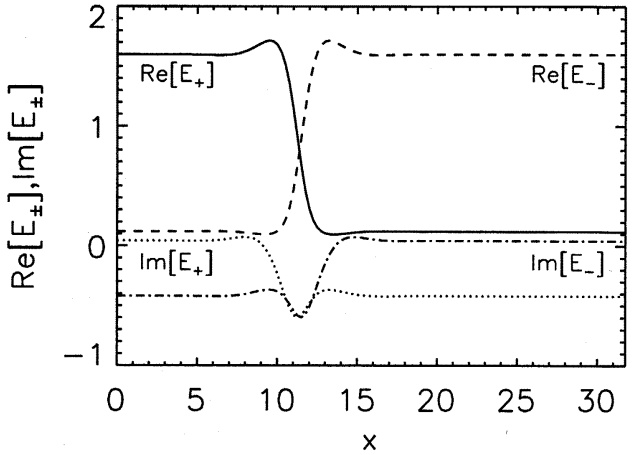

Fig. 1. Profile of the $d=1$ front connecting the two equivalent homogeneous solutions of the vectorial Kerr resonator for $E_{0}=1.6$.

\section{VECTORIAL KERR RESONATORS}

The mean field equations for a self-defocusing vectorial Kerr resonator are [11], [12]

$$
\begin{aligned}
\partial_{t} E_{ \pm}=-(1-i \theta) E_{ \pm}+i \nabla^{2} & E_{ \pm}+E_{0} \\
& -\frac{1}{4} i\left[\left|E_{ \pm}\right|^{2}+\beta\left|E_{\mp}\right|^{2}\right] E_{ \pm}
\end{aligned}
$$

where $E_{ \pm}$are the circularly polarized field components, $E_{0}$ is the pump ( $x$ polarized), $\theta$ is the cavity detuning, $\nabla^{2}$ is the transverse Laplacian, and $\beta$ is related to the susceptibility tensor. In this paper, we consider $\theta=1$ and $\beta=7$. For $E_{0}<E_{t h} \approx$ 0.95 , the homogeneous symmetric solution $I_{+}=I_{-}$(where $I_{ \pm}=\left|E_{ \pm}\right|^{2}$ ) is stable [12]. At this threshold value, a $y$-polarized stripe pattern is formed. For pump values above a second threshold, $E_{0} \approx 1.5$, there is bistability between two homogeneous solutions, namely, $E_{ \pm}^{a}$ and $E_{ \pm}^{b}$, which are asymmetric $\left(I_{+}^{a} \neq I_{-}^{a}, I_{+}^{b} \neq I_{-}^{b}\right)$ and, therefore, elliptically polarized. These two solutions are equivalent in the sense that $E_{ \pm}^{a}=E_{\mp}^{b}$, so that they have the same total intensity, polarization ellipticity, and stability properties for all values of the control parameter. They differ in the orientation and in the direction of rotation of the polarization ellipse. A domain of one of these homogeneous solutions embedded in the other can, therefore, be identified as a polarization domain. The profile of a $d=1$ front connecting these two solutions for $E_{0}=1.6$ is shown in Fig. 1. The shape of these fronts has been obtained by solving numerically the $d=1$ stationary form of (4), namely

$$
\begin{aligned}
0=-(1-i \theta) E_{ \pm}(x) & +i d_{x}^{2} E_{ \pm}(x)+E_{0} \\
& -\frac{1}{4} i\left[\left|E_{ \pm}(x)\right|^{2}+\beta\left|E_{\mp}(x)\right|^{2}\right] E_{ \pm}(x)
\end{aligned}
$$

and imposing a zero derivative as the left and right boundary conditions: $d_{x} E_{ \pm}(x=0)=0$ and $d_{x} E_{ \pm}(x=L)=0$, where $L$ is the system size. From the $d=1$ front profile and following the procedure indicated in [10], we determine the value of the growth coefficient for a circular domain $\gamma$. It follows from (5) that the $d=1$ front profile depends on the pump strength $E_{0}$ and, therefore, the value of $\gamma$ will also be dependent on $E_{0}$ as shown in Fig. 2.

In the bistability region, depending on the value of $\gamma$, three dynamical regimes can be identified when increasing the control parameter $E_{0}$ (Fig. 2) [9]. They are, respectively, a regime of labyrinthine pattern formation for $E_{0}<E_{0,1}$, a regime of formation of localized structures for $E_{0,1}<E_{0}<E_{0,2}$, and 


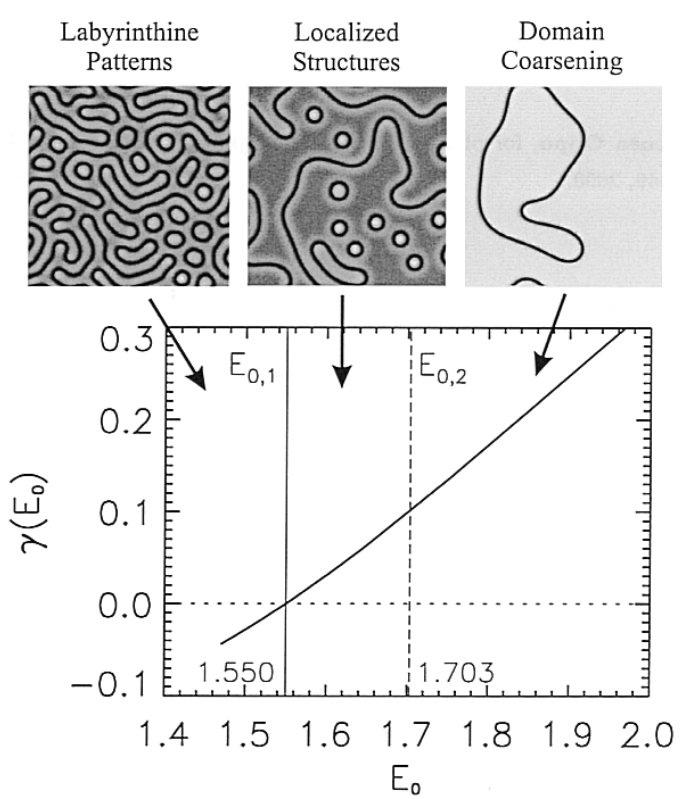

Fig. 2. Growth rate $\gamma$ versus the pump $E_{0}$ for the vectorial Kerr resonator. Vertical solid line indicates the modulational instability threshold for the flat front $E_{0,1}=1.550$. Vertical dashed line indicates the upper limit of existence of localized structures $E_{0,2}=1.703$. Upper figures show, from left to right, snapshots of typical configurations of the total intensity $I_{+}+I_{-}$in the labyrinthine localized structures and coarsening regimes.

a regime of domain coarsening for $E_{0}>E_{0,2}$ (Fig. 2). These three regimes have been experimentally observed in a four-wave mixing resonator [13] and [14]. As indicated previously, $E_{0,1}$ is the modulational instability threshold of a flat domain wall connecting the two asymmetric homogeneous states [9], [10]. $E_{0,2}$ is the limit of existence of localized structures, as will be explained below. In the regime of labyrinthine pattern formation $\left(E_{0}<E_{0,1}\right) \gamma<0$, a flat domain wall is modulationally unstable and a circular domain grows until its boundary breaks up because of the modulational instability. This behavior indicates that the system prefers to have the longest possible domain walls or, equivalently, the largest possible curvature.

For $E_{0}>E_{0,1}, \gamma>0$ and very large circular domains progressively shrink. Away from the flat wall modulational instability threshold $\left(E_{0}>1.57\right)$, however, but before $E_{0,2}$, the shrinking is halted by the formation of DRCS. These structures owe their stability to the interaction of the oscillatory tails of the domain walls, the interaction being stronger the larger the amplitude of the local oscillation. For a certain class of $d=1$ systems, and under some approximations, it has been shown that the interaction of two distant domain walls can be described by a potential with several wells which become progressively deeper the shorter the distances between the domain walls [14]. The wells of the interaction potential are located at the distances where the extrema of the oscillations of the tails overlap with each other. Our nonlinear optical systems do not belong to this class of systems, but equilibrium distances in $d=1$ and $d=2$ are also found whenever the extrema of the local oscillations of the domain walls overlap with each other [8]. For the vectorial Kerr resonator, we found that only locking at the first maxima of the oscillations is effective in counterbalancing the shrinking, while for the DOPO, as will be shown in the next section, there

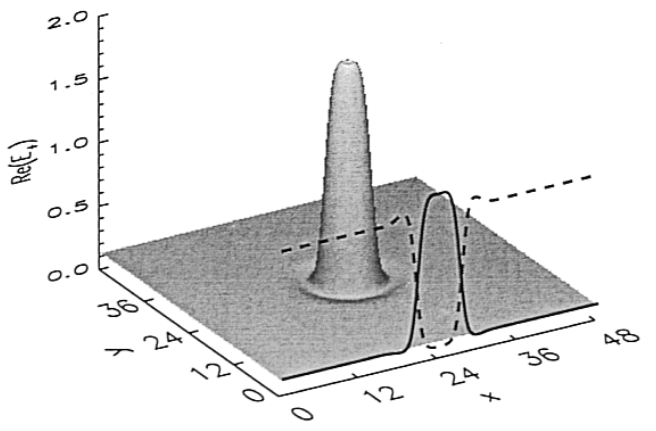

Fig. 3. DRCS of the vectorial Kerr resonator model for $E_{0}=1.6$ and the other parameters specified in the text. Vertical variable is the real part of the $E_{+}$ field. Dashed line represents the central section of the real part of the $E_{-}$field.

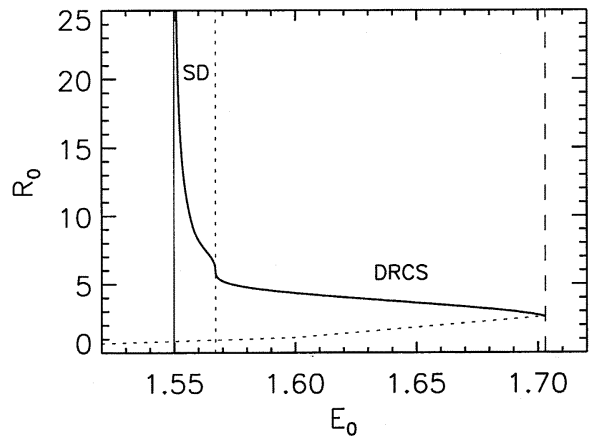

Fig. 4. Radius of the cavity solitons as a function of the pump parameter $E_{0}$ for the vectorial Kerr resonator. Solid (dotted) lines correspond to stable (unstable) cavity solitons. Vertical solid line indicates $E_{0,1}$. Vertical dashed line indicates $E_{0,2}$. Vertical dotted line indicates $E_{0,3}$, the transition from DRCS to stable droplet (see text).

are several equilibrium distances. Fig. 3 shows a typical cross section of a DRCS for $E_{0}=1.6$. It has been calculated by numerically solving the stationary radial equation [8]

$$
\begin{aligned}
0=-(1-i \theta) E_{ \pm}(r) & +i\left(d_{r}^{2}+\frac{1}{r} d_{r}\right) E_{ \pm}(r)+E_{0} \\
& -\frac{1}{4} i\left[\left|E_{ \pm}(r)\right|^{2}+\beta\left|E_{\mp}(r)\right|^{2}\right] E_{ \pm}(r)
\end{aligned}
$$

with zero derivative at the boundaries: $d_{r} E_{ \pm}(r=0)=0$ and $d_{r} E_{ \pm}(r=L)=0$, where $L$ is the system size (which we take much larger than the size of the soliton). The vertical variable in Fig. 3 is the real part of the circularly polarized $E_{+}$field. The name DRCS refers, instead, to the form of the total intensity ( + and - circularly polarized fields) as shown, for example, in the upper panels of Fig. 2. We note that, at difference with the DOPO, the total intensity on the dark ring is not zero, but we use the name DRCS because they are the same kind of structures and the ring around the central peak takes the lowest value of the total intensity.

There is an important difference between the $d=1$ and $d=2$ cases. In $d=2$, increasing the pump there is a threshold $\left(E_{0}=E_{0,2}\right.$ (Fig. 2 and 4) for the interaction of the local oscillations to counterbalance the shrinking of the circular domains due to the local curvature of the walls. Fig. 4 shows the size of the cavity solitons in $d=2$. It has been calculated by solving (6). The solid line corresponds to the stable DRCS and stable droplet while the dotted line corresponds to unstable localized 


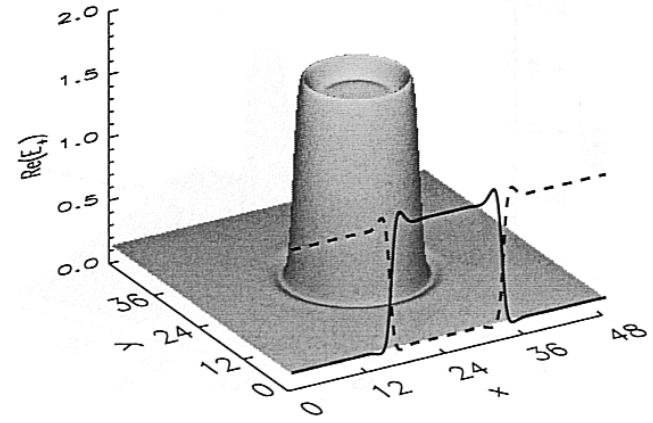

Fig. 5. Stable droplet of the vectorial Kerr resonator model for $E_{0}=1.55238$ and the other parameters specified in the text. Vertical variable is the real part of the $E_{+}$field. Dashed line represents the central section of the real part of the $E_{-}$field.

structures which have a shape similar to that of the stable DRCS. The threshold for existence of DRCS is marked in the diagram of Fig. 2 as $E_{0,2}$. It may appear counterintuitive that DRCS lose their stability for increasing pump intensities where one would expect diffraction to be more effective on the local domain walls. The amplitude of the local oscillations at the tails of the domain walls, however, has a complex dependence on the parameters of the system and decreases for increasing input energies. Furthermore, it is clear from Fig. 2 that the coefficient $\gamma$ grows with the pump; therefore, the shrinking force due to front curvature becomes more important, overcoming the interaction of the tails at $E_{0}=E_{0,2}$. In terms of stationary solutions, this threshold corresponds to a saddle-node bifurcation where the stable and the unstable branches of the DRCS collide (Fig. 4). No cavity solitons are observed for pump values larger than $E_{0,2}$, indicating that this is the threshold for domain coarsening.

We focus now on the region close to the modulational instability of the flat domain wall but for $E_{0}>E_{0,1}$. In agreement with the theory of the previous section, close to the point $E_{0,1}$ where $\gamma$ vanishes, nonlinear corrections to (1) must be considered [10]. The derivation of the amplitude equation (2), carried out for a general system in [10], yields $c_{1}=0.591$ and $c_{3}=-0.393$ for the case considered here. Since $c_{3}$ is negative, we expect a region of stability of stable droplet. These are indeed found in numerical simulations as Fig. 4 and Fig. 5 clearly display. The stable droplets are found exactly in the same way as the DRCS, namely, by solving (6) as indicated above. As expected, the radius of the stable droplet quickly diverges to infinity the closer we move to $E_{0,1}$. In the vectorial Kerr resonator, the stable droplet is in fact an elliptically polarized domain embedded in a background with opposite elliptical polarization and, therefore, it constitutes a cavity polarization soliton.

We have checked that the stable-droplet cavity soliton found in the vectorial Kerr resonator model has the universal properties suggested in Section II. At the center of the stable droplet, the field takes the value of one of the homogeneous solutions. Depending on the value of the pump, the radius of the stable droplet can be extremely large. In fact, the radius diverges at $E_{0,1}$ as $R_{0} \approx 1 / \sqrt{E_{0}-E_{0,1}}$. Fig. 6 shows a comparison of $1 / R_{0}^{2}$ between the theory of (3) (dotted line in Fig. 6) and the numerical simulations (crosses) in the vicinity of $E_{0,1}$ (vertical solid line) where stable droplets are large. The agreement is ex-

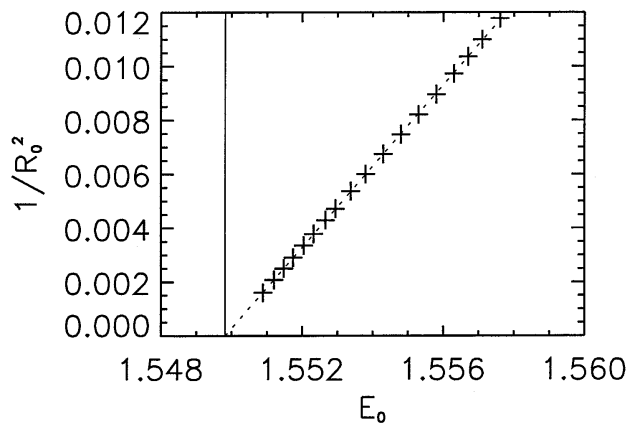

Fig. 6. Linear dependence of $1 / R_{0}^{2}$ with the control parameter $E_{0}$ for the stable droplet in the vectorial Kerr resonator. Dotted line corresponds to (3) with $c_{1}=0.591$ and $c_{3}=-0.393$. Crosses are from the numerical determination of the stable droplet as steady states of (4)

cellent, thus demonstrating that the stable droplet are a universal feature of systems with modulational instability of the flat front.

Moving now in the direction of increasing $E_{0}$, we find that the stable-droplet stable branch has a change of behavior at $E_{0}=E_{0,3}$ (Fig. 4). This particular point corresponds to the value of the pump for which the interaction of the tails becomes of the same order as the nonlinear correction of (2). We note that the transition from DRCS to stable droplet is continuous and that the stable droplet nucleates out of the DRCS. Such a transition is, however, marked by a sudden change in the size of the cavity soliton. While the radius of the DRCS changes very little with decreasing control parameter $E_{0}$, the radius of the stable droplet changes rapidly with $\left(E_{0}-E_{0,1}\right)$ as shown in (3). For $E_{0,3}<E_{0}<E_{0,2}$, the interaction of the oscillatory tails is dominant and the cavity soliton is a DRCS characterized by a larger intensity at the center than in the surrounding background (Fig. 3). For $E_{0,1}<E_{0}<E_{0,3}$, the nonlinear curvature effects (including the growth which leads to the stable droplet) dominate over the interaction of the oscillatory tails and a stable stationary circular domain wall is formed. We note here that unlike the DOPO case discussed in Section IV, the stable droplet emerges directly from the single-peak DRCS and that there is no bistability between any DRCS and stable droplet.

\section{DOPO}

The second nonlinear optical device that we consider is the phase-matched DOPO where both pump $A_{0}$ and signal $A_{1}$ fields are resonant. The mean field equations are [15]

$$
\begin{aligned}
& \partial_{t} A_{0}=\Gamma\left[-A_{0}+E_{0}-{ }_{1}^{2}\right]+\frac{i a}{2} \nabla^{2} A_{0} \\
& \partial_{t} A_{1}=-A_{1}-i \Delta_{1} A_{1}+A_{0} A_{1}^{*}+i a \nabla^{2} A_{1}
\end{aligned}
$$

where $\Gamma$ is the ratio between the pump and signal cavity decay rates, $E_{0}$ is the amplitude of the external pump field (our control parameter), $\Delta_{1}$ is the signal detuning and $a$ is the diffraction parameter. In [7] and [8], DRCS have been found for $\Delta_{1}=0$ (resonant case) and for $\Delta_{1}>0$. In order to find a modulational instability of the flat front, however, we need to turn our attention to negative signal detunings and we choose $\Delta_{1}=-1$. Moreover, it is possible to show that the modulational instability of the flat front approaches the signal generation threshold from above in the limit of high $\Gamma$ in a way analogous to what is ob- 


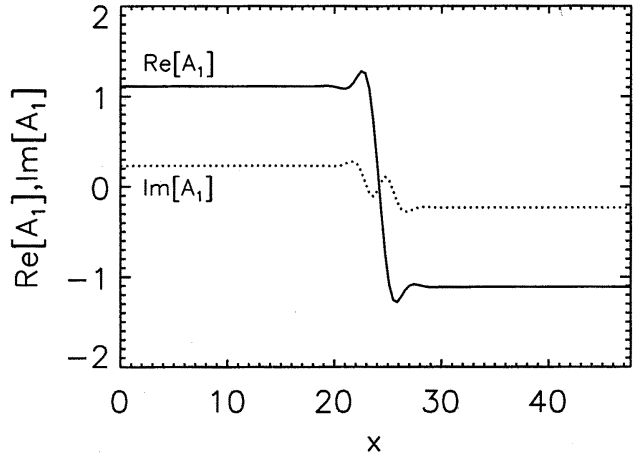

Fig. 7. Profile of the 1-D front connecting the two equivalent homogeneous solutions of the DOPO for $E_{0}=2.5$.

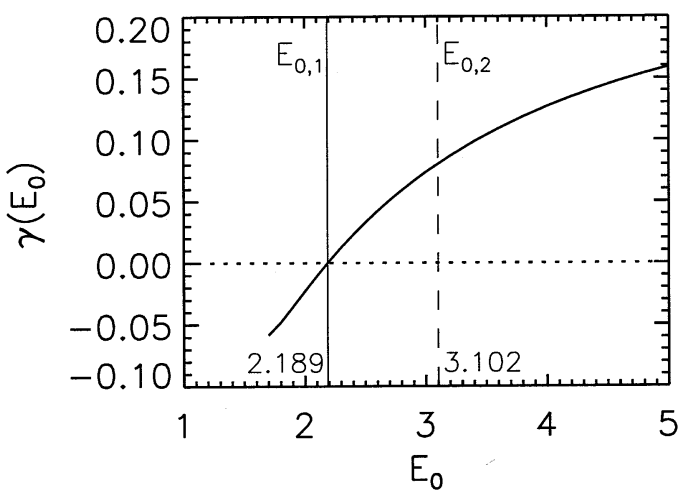

Fig. 8. Growth rate $\gamma$ for the DOPO equations (7). Vertical solid line indicates $E_{0,1}$. Vertical dashed line indicates $E_{0,2}$.

served for the thresholds of DRCS and pattern formation for positive and zero signal detunings [8]. We then select $\Gamma=6$ and $a=0.5$, which allow for the analysis of the transition from DRCS to stable droplet at parameter values which are experimentally accessible. For $0<E_{0}<1$, the zero homogeneous solution is stable. Above the $E_{0}=1$ threshold, a stripe intensity pattern is formed [15]. For pump values above a second threshold, $E_{0} \approx 1.56$, there is multistability between two nonzero homogeneous solutions and several (regular or irregular) spatially modulated solutions. The latter cease to exist at the threshold of labyrinthine patterns, i.e., the modulational instability of the flat front connecting the two homogeneous solutions, which occurs at $E_{0,1}=2.189$. The two homogeneous solutions have the same intensity and stability properties for all values of the control parameter. They only differ by a $\pi$ phase shift in the signal field $A_{1}$. The profile of a $d=1$ front connecting these two homogeneous solutions is shown in Fig. 7. As with the vectorial Kerr resonator, the front profile has been calculated by solving numerically the $d=1$ stationary form of (7)

$$
\begin{aligned}
& 0=\Gamma\left[-A_{0}(x)+E_{0}-A_{1}^{2}(x)\right]+\frac{i a}{2} d_{x}^{2} A_{0}(x) \\
& 0=-A_{1}(x)-i \Delta_{1} A_{1}(x)+A_{0}(x) A_{1}^{*}(x)+i a d_{x}^{2} A_{1}(x) .
\end{aligned}
$$

Fig. 8 shows the behavior of the growth rate for a circular domain $\gamma\left(E_{0}\right)$ for the DOPO calculated as indicated in [10] from the $d=1$ front profile. As for the vectorial Kerr resonator case,

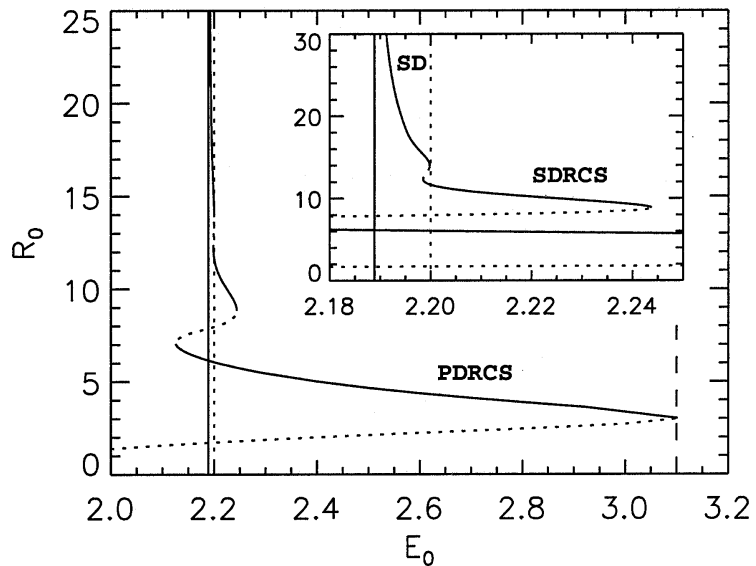

Fig. 9. Radius of the cavity solitons as a function of the pump parameter $E_{0}$ for the DOPO. Solid (dotted) lines correspond to stable (unstable) cavity solitons. Primary (secondary) DRCS are labeled as PDRCS (SDRCS). Vertical solid line indicates $E_{0,1}$. Vertical dashed line indicates $E_{0,2}$. Vertical dotted line indicates $E_{0,3}$, the transition from DRCS to stable droplet. Inset shows a closeup of this transition.

three dynamical regimes can be identified. For $E_{0}>E_{0,2}$, there is a regime of domain coarsening and no cavity solitons. For $E_{0,1}<E<E_{0,2}$ asymptotic cavity solitons exist, either DRCS or stable droplet. Finally, for $E_{0}<E_{0,1}$, labyrinthine patterns appear. We note here a difference with the vectorial Kerr resonator case. In this region, the interaction of the tails of the domain walls between the stable homogeneous solutions is still strong enough to stabilize a single peak (primary) DRCS corresponding to the locking of the first maxima of the oscillatory tails. This is clearly visible in Fig. 9, where one branch of the DRCS extends well below the modulational instability threshold at $E_{0,1}$ where labyrinthine patterns exist. Note that the $d=2$ cavity solitons (of the stable droplet or DRCS type) and their stability have been calculated by solving the stationary radial equations

$$
\begin{aligned}
0= & \Gamma\left[-A_{0}(x)+E_{0}-A_{1}^{2}(x)\right]+\frac{i a}{2}\left(d_{r}^{2}+\frac{1}{r} d_{r}\right) A_{0}(x) \\
0= & -A_{1}(x)-i \Delta_{1} A_{1}(x)+A_{0}(x) A_{1}^{*}(x) \\
& +i a\left(d_{r}^{2}+\frac{1}{r} d_{r}\right) A_{1}(x)
\end{aligned}
$$

with the numerical methods described in [8].

Since the stable droplets are always on the side opposite to the labyrinths, stable droplets for the DOPO with these parameters cannot nucleate out of the single-peak DRCS. It has been shown in [8] that the single-peak DRCS, corresponding to the locking of the first maxima, is just one of several solitons whose stability is due to the interactions of the tails of the domain walls. For example, a secondary DRCS with a central trough, corresponding to the locking of the first minima, is also stable at resonance. We have also located such secondary DRCS structures in the vicinity of the modulational instability of the flat front. The uppermost solid line on the right side of $E_{0,3}$ in Fig. 9 gives the radius of the stable DRCS displayed in Fig. 10. Unlike the DOPO at resonance $\left(\Delta_{1}=0\right)$ considered in [7] and [8], the unstable branch of the secondary (trough) DRCS merges with the stable branch of the primary (single-peak) DRCS at around $E_{0} \sim 2.126$. We explain this fact by noting that $\gamma\left(E_{0}\right)$ of (1) 


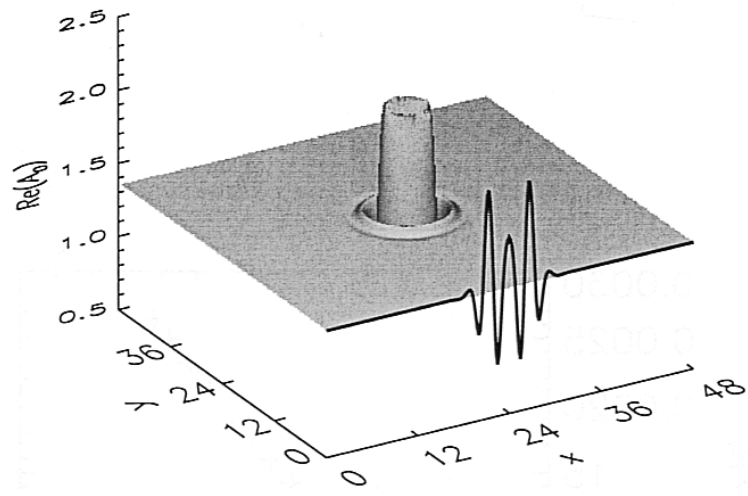

(a)

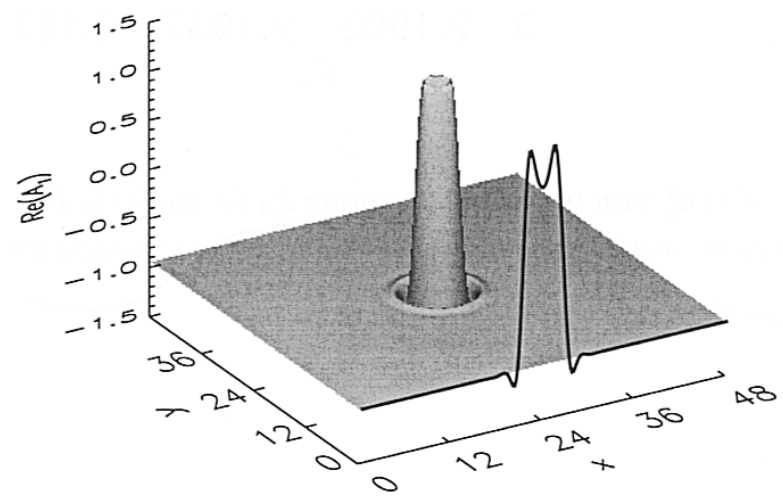

(b)

Fig. 10. (a) Real part of the pump and (b) signal fields of a secondary (trough) DRCS for the DOPO at $E_{0}=2.21$. Solid lines are the central sections of the three-dimensional (3-D) plots.

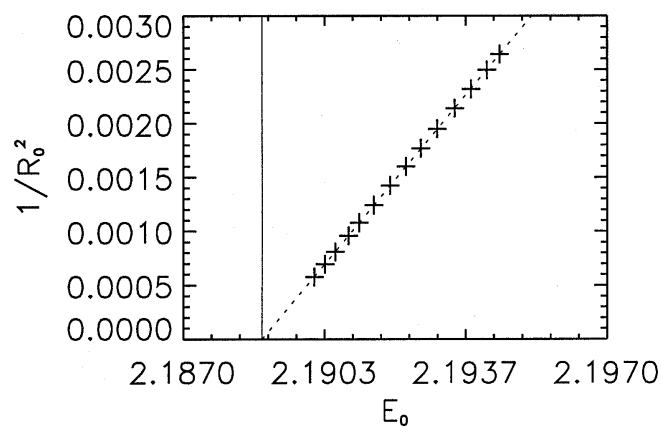

Fig. 11. Linear dependence of $1 / R_{0}^{2}$ with the control parameter $E_{0}$ for the stable droplet in DOPO. Dotted line corresponds to (3) with $c_{1}=0.115$ and $c_{3}=-0.244$. Crosses are from the numerical determination of the stable droplet as steady states of (7).

increases with $E_{0}$ in the case described here (Fig. 9) and instead decreases with $E_{0}$ at resonance.

The secondary DRCS loses stability just after the $E_{0,3}$ line of Fig. 9 when decreasing $E_{0}$. The unstable branch of this saddle-node bifurcation is then linked to the stable branch of the stable droplet which start to inflate at $E_{0,3}$. Again, one observes a sudden change in the radius of the cavity solitons when switching from DRCS to stable droplets. The radius of the stable droplet then quickly grows with the parameter $E_{0}$ approaching the modulation instability of the stable front, as clearly displayed in Fig. 9. As shown in Fig. 11, stable droplets in the DOPO again satisfy the general equation (3) with $c_{1}=0.115$ and $c_{3}=-0.244$, where the value of these

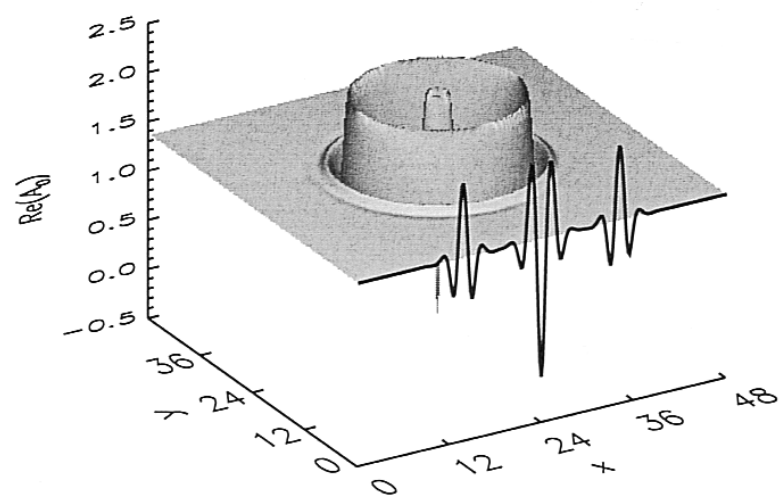

(a)

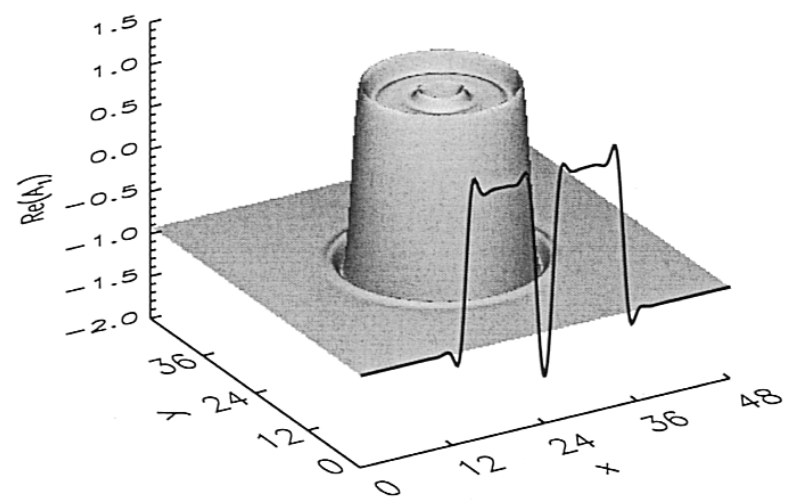

(b)

Fig. 12. Primary (single-peak) DRCS at the center of a stable droplet for the DOPO at $E_{0}=2.19015$. Both cavity solitons taken separately are stable in this parameter region. (a) Real part of the pump field. (b) Real part of the signal field. Solid lines are the central sections of the 3-D plots.

coefficients is obtained from the derivation of the amplitude equation (2) following the procedure indicated in [10]. One important aspect of the stable droplet in the DOPO system is that stable droplets are now bichromatic since they are visible in both pump and signal fields. Interesting correlations of fluctuations between spatial solitons at the fundamental and second harmonic frequencies have recently been reported [16]. We are now extending these studies to parametric downconversion in cavities where the DRCS and stable droplet are good candidates for their observation.

In the entire regime of the existence of stable droplets in the DOPO, we observe bistability of stable droplets and primary (single-peak) DRCS (Fig. 9). We show in Fig. 12 an interesting composite cavity soliton which is a natural consequence of this bistability. A primary (single-peak) DRCS is stable at the center of a large stable droplet at $E_{0}=2.19015$.

\section{CONCLUSION}

We have demonstrated within the context of a general theory that in two different nonlinear optical systems there exist stable circular domains. For the vectorial Kerr resonator, these domains are in fact polarization domains, cavity polarization solitons, while for the optical parametric oscillator, they are domains of opposite phase. This offers two different possibilities for addressing and manipulating cavity solitons. 
We have also shown that stable droplets nucleate out of DRCS but can coexist with DRCS in the parameter space. This fact may be exploited in increasing the information capacity of these devices by using the stable droplet and DRCS to encode separate information.

\section{ACKNOWLEDGMENT}

The authors would like to thank G. K. Harkness, W. J. Firth, and E. Hernández-García for useful discussions.

\section{REFERENCES}

[1] "Special issue on solitons," Opt. Photon. News, vol. 13, no. 2, pp. 27-76, 2002.

[2] W. J. Firth and A. J. Scroggie, "Optical bullet holes: robust controllable localized states of a nonlinear cavity," Phys. Rev. Lett., vol. 76, pp. 1623-1626, 1996.

[3] W. J. Firth and G. K. Harkness, "Cavity solitons," Asian J. Phys., vol. 7, no. 3, pp. 665-677, 1998.

[4] S. Trillo, M. Haelterman, and A. Sheppard, Opt. Lett., vol. 22, p. 970, 1997.

[5] K. Ouchi and H. Fujisaka, "Phase ordering kinetics in the Swift-Hohenberg equation," Phys. Rev. E, vol. 54, pp. 3895-3898, 1996.

[6] K. Staliunas and V. J. Sanchez-Morcillo, "Spatial-localized structures in degenerate optical parametric oscillators," Phys. Rev. A, vol. 57, pp. 1454-1457, 1998.

[7] G.-L. Oppo, A. J. Scroggie, and W. J. Firth, J. Opt. B, vol. 1, p. 133, 1999.

[8] - "Characterization, dynamics and stabilization of diffractive domain walls and dark-ring cavity solitons in parametric oscillators," Phys. Rev. E, vol. 63, p. $066209,2001$.

[9] R. Gallego, M. San Miguel, and R. Toral, "Self-similar doamin growth, localized structures, and labyrinthine patterns in vectorial Kerr resonators," Phys. Rev. E, vol. 61, pp. 2241-2244, 2000.

[10] D. Gomila, P. Colet, G.-L. Oppo, and M. San Miguel, "Stable droplets and growth laws close to the modulational instability of a domain wall," Phys. Rev. Lett., vol. 87, p. 194 101, 2001.

[11] J. B. Geddes, J. V. Moloney, E. M. Wright, and W. J. Firth, Opt. Commun., vol. 111, p. 623, 1994

[12] M. Hoyuelos, P. Colet, M. San Miguel, and D. Walgraef, "Polarization patterns in Kerr media," Phys. Rev. E, vol. 58, pp. 2292-3007, 1998

[13] V. B. Taranenko, K. Staliunas, and C. O. Weiss, "Pattern formation and localized structures in degenerate optical parametric mixing," Phys. Rev. Lett., vol. 81, pp. 2236-2239, 1998.

[14] P. Coullet, C. Elphick, and D. Repaux, "Nature of spatial chaos," Phys. Rev. Lett., vol. 65, pp. 431-434, 1987.

[15] G.-L. Oppo, M. Brambilla, and L. A. Lugiato, "Formation and evolution of roll patterns in optical parametric oscillators," Phys. Rev. A, vol. 49, pp. 2028-2032, 1994.

[16] N. Treps and C. Fabre, "Transverse distribution of quantum fluctuations and correlations in spatial solitons," Phys. Rev. A, vol. 62, p. 033816 , 2000.

[17] C. O. Weiss, M. Vaupel, K. Staliunas, G. Slekys, and V. B. Taranenko, "Solitons and vortices in lasers," Appl. Phys. B, vol. 68, p. 151, 1999.

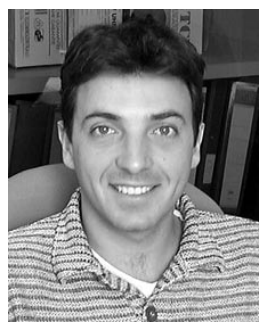

Damià Gomila was born in Maó, Menorca, Spain, in 1976, and graduated in physics from the University of Barcelona, Barcelona, Spain, in 1998. He is currently working toward the Ph.D. degree at the Institut Mediterrani d'Estudis Avançats (IMEDEA), Palma de Mallorca, Spain.

His research topics are in pattern formation, nonlinear science, and statistical mechanics. He has been studying the dynamics of extended and localized spatial structures in the transverse cross section of light beams interacting with nonlinear optical media.

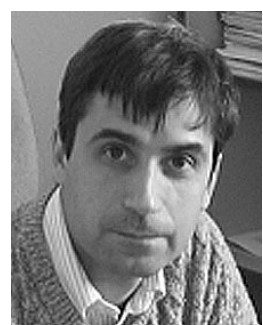

Pere Colet was born in Barcelona, Spain, in 1964. He received the M.Sc. degree in physics from the University of Barcelon, Barcelona, Spain, in 1987, and the $\mathrm{Ph} . \mathrm{D}$. degree from the Universitat de les Illes Balears, Palma de Mallorca, Spain, in 1991.

In 1991, he became a Teaching Assistant in the Department of Physics, Universitat de les Illes Balears. During 1991-1994, he was a postdoctoral Fulbright Fellow at the School of Physics, Georgia Institute of Technology, Atlanta. In October 1994, he joined the Department of Physics, Universitat de les Illes Balears, where he has held a permanent research position since May 1995 at the Spanish Consejo Superior de Investigaciones Cientificas. He has coauthored 55 journal papers as well as 20 other scientific publications. His research interests include fluctuations and nonlinear dynamics of semiconductor lasers, synchronization of chaotic lasers and encoded communications, synchronization of coupled nonlinear oscillators, pattern formation and quantum fluctuations in nonlinear optical cavities, and dynamics of localized structures.

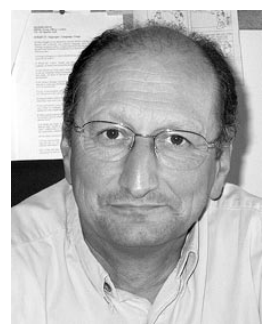

Maxi San Miguel was born in Barcelona, Spain, in 1952. He received the M.Sc. and the Ph.D. degrees in theoretical physics from the University of Barcelona, in 1974 and 1978, respectively.

He was a Research Associate at the Physics Department, Temple University, Philadelphia, PA, during 1979-1980 and in 1984. From 1980 to 1986, he was with the Theoretical Physics Department, University of Barcelona. Since 1986, he has been a Professor of Physics at the University of the Balearic Islands, Spain. He has also been a Visiting Professor at the University La Sapienza, Rome, Italy. His current research interests include modeling of fluctuations and nonlinear properties of semiconductor lasers.

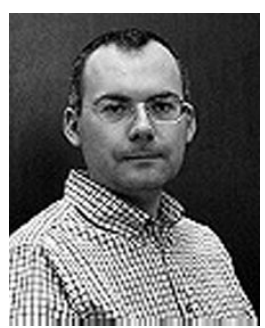

Andrew J. Scroggie was born in Glasgow, Scotland, U.K., in 1970. He received the Ph.D. degree in physics from the University of Strathclyde, Strathclyde, U.K., in 1995.

He has been a Research Assistant with the Computational Nonlinear and Quantum Optics Group, Department of Physics, University of Strathclyde, since 1995, with pivotal contributions on optical bullet holes, control of optical pattern formation, cavity solitons in optical parametric oscillators, and semiconductor microresonators. His main research interests are in the theoretical and numerical analysis of spatially extended nonlinear optical devices, generation of ultrashort optical pulses, and formation and dynamics of optical structures.

Dr. Scroggie is a member of the Institute of Physics.

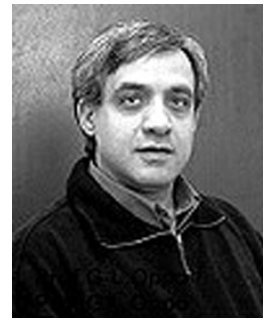

Gian-Luca Oppo was born in Modena, Italy, in 1958. He received the Ph.D. degree in physics from the University of Florence, Florence, Italy, in 1983.

He has been a Professor of computational and nonlinear physics since 1998. He has worked in Toronto, ON, Canada, and Philadelphia, PA, and joined the University of Strathclyde, Glasgow, U.K., in 1989. Together with W. J. Firth, he set up the Computational Nonlinear Optics Group for the theoretical and numerical analysis of spatial and temporal structures in lasers and other nonlinear optical devices. His main interests in optics include control of pattern formation, nonlinear dynamics of optical devices, quantum images, spatial solitons, short pulses in optical parametric oscillators, coherences in atomic emissions, and absorptions. He is coordinating, with S. M. Barnett, the European Network Quantum Structures. 